\title{
Erratum
}

\section{Antioxidant enzymes in oligodendroglial brain tumors: association with proliferation, apoptotic activity and survival}

Sally Järvelä, Helena Bragge, Niina Paunu, Timo Järvelä, Leo Paljärvi, Hannu Kalimo, Pauli Helén, Vuokko Kinnula, Ylermi Soini and Hannu Haapasalo

Journal of Neuro-Oncology

DOI 10.1007/s11060-005-9030-z

The author names were misrepresented on the title page of the original. 